\title{
Singular solutions to the Seiberg-Witten and Freund equations on flat space from an iterative method
}

\author{
Ricardo A. Mosna \\ Instituto de Matemática, Estatística e Computação Científica, \\ Universidade Estadual de Campinas, C.P. 6065, 13083-859, Campinas, SP, Brazil.
}

(Dated: September 14, 2018)

\begin{abstract}
Although it is well known that the Seiberg-Witten equations do not admit nontrivial $L^{2}$ solutions in flat space, singular solutions to them have been previously exhibited - either in $\mathbb{R}^{3}$ or in the dimensionally reduced spaces $\mathbb{R}^{2}$ and $\mathbb{R}^{1}$ - which have physical interest. In this work, we employ an extension of the Hopf fibration to obtain an iterative procedure to generate particular singular solutions to the Seiberg-Witten and Freund equations on flat space. Examples of solutions obtained by such method are presented and briefly discussed.
\end{abstract}

PACS numbers: 11.10.Lm, 02.40.-k, 11.15.-q

\section{INTRODUCTION}

Given a physical system defined on a configuration space $M$, there are various instances where it is useful to employ (extensions of) fibrations $P \rightarrow M$ to lift the corresponding equations of motion from $M$ to $P$. For instance, the natural extension of the Hopf fibration $S^{3} \rightarrow S^{2}$ to $\mathbb{R}^{4} \rightarrow \mathbb{R}^{3}$ (defining the so-called Kustaanheimo-Stiefel transformation [1, 2]) can be used to map the Kepler problem in $\mathbb{R}^{3}$ to a harmonic oscillator problem in $\mathbb{R}^{4}$. This construction has been recurrently employed to regularize and calculate orbits of celestial objects, besides giving rise to various applications in atomic physics (see, e.g., 3 and references therein). In this work, we apply this idea to the case when $M$, instead of representing the configuration space of a particle, is the target space of a given field theory. Specifically, we show that by lifting the equations of magnetostatics (in the sense above), it is possible to obtain the Seiberg-Witten equations (SWE) on $\mathbb{R}^{3}$ provided that a certain constraint is imposed on the resulting fields. Moreover, we show that such constraint naturally gives rise to an iterative method to generate particular solutions to the SWE and Freund equations on $\mathbb{R}^{3}$ and its dimensionally reduced spaces.

It should be kept in mind that the SWE do not admit nontrivial $L^{2}$ solutions in flat space 44 (the same is not true for the Freund equations [ 5 ]). However, singular solutions to the SWE in flat space do exist [5, [6], with physical interest. Another point to be emphasized is that the lifting procedure considered here (Section III) is not new since it is implicit in the pioneer work of Loss and Yau on zero modes of the three-dimensional Dirac operator [7] (it is also known that, by applying the Kustaanheimo-Stiefel transformation to the vector potential coupled to a Dirac spinor, one recovers the ansatz of Loss and Yau [8]). In fact, our formulae for the relevant Abelian potential $A_{k}$ and wavefunction $|\psi\rangle$ are the same as those of 7 ], where $A_{k}$ and $|\psi\rangle$ are given in terms of a generating vector field, but with the differences that, in our case, such generating vector field satisfies a certain constraint and that, due to the singular nature of the present problem, we do not demand that the associated fields be square integrable.

It is precisely such constraint that gives rise, in our approach, to the aforementioned iterative method to the SWE and Freund equations on $\mathbb{R}^{3}$. This is considered in Section $\amalg$ where we also show that application of such method recovers some known solutions to the SWE and Freund equations and yields, to the best of our knowledge, previously unnoticed solutions to the SWE. In particular, we obtain an axisymmetric singular solution to the SWE on $\mathbb{R}^{3}$. We conclude by presenting some final remarks in Section IV

\section{LIFTING THE MAGNETOSTATICS EQUATIONS}

We start from the equations of magnetostatics,

$$
\begin{aligned}
\nabla \cdot \boldsymbol{H} & =0, \\
\nabla \times \boldsymbol{H} & =\boldsymbol{J},
\end{aligned}
$$

*Electronic address: mosna@ime.unicamp.br 
where $\boldsymbol{J}$ is the steady current associated with the magnetic field $\boldsymbol{H}$ (we use Heaviside-Lorentz units with c=1). Let $|\psi\rangle$ be a two-component spinor such that

$$
H^{k}=\left\langle\psi\left|\sigma^{k}\right| \psi\right\rangle, \quad k=1,2,3
$$

where $\sigma^{1}, \sigma^{2}, \sigma^{3}$ are the Pauli matrices. For each $\boldsymbol{r} \in \mathbb{R}^{3}, \boldsymbol{H}(\boldsymbol{r})$ can be formally regarded as the "polarization vector" or "spin density" associated with $|\psi(\boldsymbol{r})\rangle \in \mathbb{C}^{2}$, as in quantum mechanics textbooks [9]. The general solution of Eq. (2) for $|\psi\rangle$ in terms of $\boldsymbol{H}$ is given by ${ }^{1}$

$$
|\psi\rangle=e^{-i \chi} \frac{1}{\sqrt{2\left(H+H^{3}\right)}}\left(\begin{array}{c}
H+H^{3} \\
H^{1}+i H^{2}
\end{array}\right),
$$

where $H=\|\boldsymbol{H}\|$ and $e^{-i \chi}$ is an arbitrary phase factor. ${ }^{2}$

Before transferring the dynamics (Eqs. (1) from $\boldsymbol{H}$ to $|\psi\rangle$, we briefly consider the geometry underlying Eqs. (2) and (3). Let $S_{a}^{n}$ denote the $n$-sphere of radius $a$ in $\mathbb{R}^{n+1}$, and consider the map $\pi_{a}: S_{a}^{3} \rightarrow S_{a^{2}}^{2}$ taking a two-component spinor $|\phi\rangle \in S_{a}^{3} \subset \mathbb{C}^{2}$ into the vector $s \in S_{a^{2}}^{2}$ with components $s^{k}=\left\langle\phi\left|\sigma^{k}\right| \phi\right\rangle{ }^{3}$ This defines a principal fiber bundle $U(1) \cdots S_{a}^{3} \rightarrow S_{a^{2}}^{2}$ which is essentially the first Hopf bundle (where one usually takes $a=1$ ) [10]. More generally, one can drop the requirement that $|\phi\rangle$ belongs to a sphere of fixed radius and consider the map $\pi: \mathbb{R}^{4} \rightarrow \mathbb{R}^{3}$ taking $|\phi\rangle \in \mathbb{C}^{2} \cong \mathbb{R}^{4}$ into $s^{k}=\left\langle\phi\left|\sigma^{k}\right| \phi\right\rangle$. In this way, $\pi$ is a natural extension of the Hopf map $S^{3} \rightarrow S^{2}$ to $\mathbb{R}^{4} \rightarrow \mathbb{R}^{3}$, in which each sphere of radius $a>0$ in $\mathbb{R}^{4}$ is mapped into the sphere $S_{a^{2}}^{2} \subset \mathbb{R}^{3}$, and the origin of $\mathbb{R}^{4}$ is mapped into the origin of $\mathbb{R}^{3}$. Such map defines the so-called Kustaanheimo-Stiefel transformation [1, 2] (this no longer gives rise to a principal fiber bundle, since the fiber over the origin is just a point). Note that, in our case, Eq. (2) defines a Kustaanheimo-Stiefel transformation on the corresponding target spaces, relating, for each $\boldsymbol{r}$, the vector $\boldsymbol{H}(\boldsymbol{r}) \in \mathbb{R}^{3}$ to the spinor $|\psi(\boldsymbol{r})\rangle \in \mathbb{C}^{2} \cong \mathbb{R}^{4}$. We also note that Eq. (3) yields, for each fixed $\boldsymbol{r}$, a local section of the bundle $U(1) \cdots S_{H}^{3} \rightarrow S_{H^{2}}^{2}$ over $S_{H^{2}}^{2} \backslash\{$ south pole $\}$. A related local section over $S_{H^{2}}^{2} \backslash\{$ north pole $\}$ can be similarly obtained.

Going back to Eq. (3), it is easy to see that the density matrix associated with $|\psi\rangle$ is given by

$$
|\psi\rangle\langle\psi|=\frac{1}{2}\left(H \mathbb{1}+H^{k} \sigma_{k}\right)
$$

where $\mathbb{1}$ is the identity $2 \times 2$ matrix (notice that we are working with Cartesian coordinates in Euclidean flat space, so that indices can be freely raised and lowered). For what follows, it is useful to define the following matrix-valued functions:

$$
\begin{aligned}
\mathrm{H}(\boldsymbol{r}) & =H^{k}(\boldsymbol{r}) \sigma_{k}, \\
\mathrm{~J}(\boldsymbol{r}) & =J^{k}(\boldsymbol{r}) \sigma_{k} .
\end{aligned}
$$

Then, it is easily seen that Eqs. (1) can be equivalently written, in terms of $\mathrm{H}$ and J, as

$$
\partial \mathrm{H}=i \mathrm{~J}
$$

where $\partial=\sigma^{k} \partial_{k}$ (this follows at once from the relationship $\sigma^{i} \sigma^{j}=\delta^{i j} \mathbb{1}+i \epsilon^{i j k} \sigma_{k}$ satisfied by the Pauli matrices, where $\epsilon^{i j k}$ is the totally antisymmetric symbol with $\epsilon^{123}=1$ ).

We now transfer the dynamics defined by Eqs. (1) from $\boldsymbol{H}$ to $|\psi\rangle$. From Eq. (4):

$$
\mathrm{H}=2|\psi\rangle\langle\psi|-H \mathbb{1},
$$

which leads, upon substitution into Eq. (6), to

$$
\sigma^{k}\left[\partial_{k}|\psi\rangle\langle\psi|+| \psi\rangle \partial_{k}\langle\psi|-\frac{1}{2} \partial_{k} H-\frac{i}{2} J_{k}\right]=0 .
$$

\footnotetext{
${ }^{1}$ In spherical coordinates, Eq. (3) assumes the familiar form $|\psi\rangle=e^{-i \chi} \sqrt{H}\left(\begin{array}{c}\cos \frac{\alpha}{2} \\ \sin \frac{\alpha}{2} e^{i \beta}\end{array}\right)$, where $\boldsymbol{H}=H(\sin \alpha \cos \beta, \sin \alpha \sin \beta, \cos \alpha)$.

${ }^{2}$ It is interesting to note that this is a (trivial) application of what has been termed the inversion theorem [11], an useful result (especially in four dimensions $[12,13]$ ) when one wants to reconstruct a given spinor, apart from arbitrary phases, from its bilinear covariants.

${ }^{3}$ Here $|\phi\rangle=\left(\begin{array}{l}z_{1} \\ z_{2}\end{array}\right) \in S_{a}^{3} \subset \mathbb{C}^{2}$ means that $\left|z_{1}\right|^{2}+\left|z_{2}\right|^{2}=a^{2}$.
} 
Our aim is to obtain a differential equation governing the dynamics of $|\psi\rangle$. To that end, we right-multiply the above equation by $|\psi\rangle$ and use the fact that $\langle\psi \mid \psi\rangle=H$. This yields

$$
\sigma^{k}\left[\partial_{k}+\frac{1}{2 H} \partial_{k} H-\frac{1}{H}\left\langle\psi\left|\partial_{k}\right| \psi\right\rangle-\frac{i}{2 H} J_{k}\right]|\psi\rangle=0
$$

The term $\left\langle\psi\left|\partial_{k}\right| \psi\right\rangle$ can be computed by a straightforward calculation; it follows from Eq. (2) that

$$
\left\langle\psi\left|\partial_{k}\right| \psi\right\rangle=\frac{1}{2} \partial_{k} H+\frac{i}{2\left(H+H^{3}\right)}\left(H^{1} \partial_{k} H^{2}-H^{2} \partial_{k} H^{1}\right)-i H \partial_{k} \chi .
$$

Upon substitution into Eq. (7), this leads to

$$
\sigma^{k}\left[\partial_{k}+i\left(\partial_{k} \chi-\frac{1}{2 H\left(H+H^{3}\right)}\left(H^{1} \partial_{k} H^{2}-H^{2} \partial_{k} H^{1}\right)-\frac{1}{2 H} J_{k}\right)\right]|\psi\rangle=0
$$

Defining

$$
A_{k}:=-\frac{1}{2 H\left(H+H^{3}\right)}\left(H^{1} \partial_{k} H^{2}-H^{2} \partial_{k} H^{1}\right)-\frac{1}{2 H} J_{k},
$$

which can be fully expressed in terms of $\boldsymbol{H}$ (through Eq. (1b) as

$$
A_{k}=-\frac{1}{2 H\left(H+H^{3}\right)}\left(H^{1} \partial_{k} H^{2}-H^{2} \partial_{k} H^{1}\right)-\frac{1}{2 H}(\nabla \times \boldsymbol{H})_{k},
$$

we finally get

$$
i \sigma^{k}\left(\partial_{k}+i A_{k}+i \partial_{k} \chi\right)|\psi\rangle=0
$$

Therefore, $|\psi\rangle$ satisfies the Weyl equation ${ }^{4}$ with the Abelian potential $A_{k}$. Note that $\chi$ enters Eqs. (3) and (9) simply as a gauge parameter.

It is interesting to note that Eq. (1b) enters the derivation above merely as a bookkeeping device. In fact, Eq. (10) for $|\psi\rangle$ follows as long as $\boldsymbol{H}$ satisfies Eq. (1a), regardless of any interpretation of the right-hand side of Eq. (1b) as an external current. In any case, it should be noted that Eq. (1b) does affect the form of Eq. (10) through $A_{k}$.

An important observation for what follows is that the field strength $B_{k}$ associated with $A_{k}$,

$$
\boldsymbol{B}:=\nabla \times \boldsymbol{A}
$$

does not have to bear any relation to the magnetic field $\boldsymbol{H}$ we started with.

\section{SEIBERG-WITTEN AND FREUND EQUATIONS}

Let us summarize what has been done above. We started from the equations of magnetostatics, expressed the magnetic field $\boldsymbol{H}$ in terms of the associated spinor field $|\psi\rangle$, and then lifted the dynamics from $\boldsymbol{H}$ to $|\psi\rangle$. As a result, the following set of equations (Eqs. (2), (10), and (11)) was obtained:

$$
\begin{aligned}
\left\langle\psi\left|\sigma^{k}\right| \psi\right\rangle & =H^{k}, \\
i \sigma^{k}\left(\partial_{k}+i A_{k}\right)|\psi\rangle & =0, \\
\epsilon^{i j k} \partial_{i} A_{j} & =B^{k},
\end{aligned}
$$

where we chose to suppress the terms associated with the gauge parameter $\chi$. The SWE and Freund equations in three dimensions have been discussed in detail in [5] (see especially its equations (3.5) and (3.6)) from where we note a remarkable similarity with Eqs. (12). More precisely:

\footnotetext{
4 That is, the massless Dirac equation for (two-component) spinors representing states of definite chirality.
} 
1. Eqs. (12) are the Seiberg-Witten equations on $\mathbb{R}^{3}$ provided that $H_{k}=+B_{k}$;

2. Eqs. (12) are the Freund equations on $\mathbb{R}^{3}$ provided that $H_{k}=-B_{k}$.

Therefore, the constraint

$$
\boldsymbol{H}= \pm \boldsymbol{B}
$$

yields a natural ansatz for obtaining solutions to the Seiberg-Witten and Freund equations for $\boldsymbol{A}, \boldsymbol{B}$, and $|\psi\rangle$ on $\mathbb{R}^{3}$. Using Eq. (9), this amounts to solving

$$
\boldsymbol{H}= \pm \nabla \times\left(-\frac{1}{2 H\left(H+H^{3}\right)}\left(H^{1} \nabla H^{2}-H^{2} \nabla H^{1}\right)-\frac{1}{2 H} \nabla \times \boldsymbol{H}\right)
$$

for $\boldsymbol{H}$. This equation has been recently studied from a group-theoretical perspective in 14 to examine the Lie symmetries of the SWE and Freund equations on $\mathbb{R}^{3}$.

It is interesting to note that, given a solution of Eq. (14), with the + or - sign, respectively, one immediately obtains $|\psi\rangle, A_{k}$, and $B_{k}$ from Eqs. (3), (91), and (13):

$$
\begin{aligned}
|\psi\rangle & =\frac{1}{\sqrt{2\left(H+H^{3}\right)}}\left(\begin{array}{c}
H+H^{3} \\
H^{1}+i H^{2}
\end{array}\right), \\
\boldsymbol{A} & =-\frac{1}{2 H\left(H+H^{3}\right)}\left(H^{1} \nabla H^{2}-H^{2} \nabla H^{1}\right)-\frac{1}{2 H} \nabla \times \boldsymbol{H} \\
\boldsymbol{B} & = \pm \boldsymbol{H} .
\end{aligned}
$$

As noted in the Introduction, the above expressions for $|\psi\rangle$ and $\boldsymbol{A}$ in terms of a generating vector field (which is given, in this case, by $\boldsymbol{H}$ ) were first obtained in the study of zero modes of the massless Dirac operator in 7 ].

\section{A. An iterative procedure}

We now show how Eq. (14) can serve as a basis for an iterative procedure for obtaining $\boldsymbol{H}$, and thus $|\psi\rangle, \boldsymbol{A}$ and $\boldsymbol{B}$ satisfying the SWE or Freund equations on flat space. The procedure goes as follows. Choose an initial guess $\boldsymbol{H}_{(0)}$ for $\boldsymbol{H}$; substitute $\boldsymbol{H}_{(0)}$ into the right-hand side of Eq. (14) and consider the result as a second estimate $\boldsymbol{H}_{(1)}$ for $\boldsymbol{H}$; then substitute $\boldsymbol{H}_{(1)}$ into the right-hand side of Eq. (14), and so on. If the sequence $\boldsymbol{H}_{(k)}$ converges, its limit is a solution to Eq. (14). It is important to note that this procedure does fail in most cases, either by computational or mathematical difficulties (we come back to this point in Section [V]). Nevertheless, when it succeeds, we end up with a solution to the Seiberg-Witten or Freund equations. In the remainder of this section, we show representative results of a limited experiment in algebraic computation, performed with the software Mathematica, implementing such iterative procedure.

Example 1. Starting with $\boldsymbol{H}_{(0)}= \pm(x, y, z)$, we obtain the solution

$$
\begin{aligned}
\boldsymbol{B} & =\mp \frac{1}{2 r^{3}}(x, y, z), \\
\boldsymbol{A} & =\frac{1}{2 r(r \pm z)}(y,-x, 0) \\
|\psi\rangle & =\frac{1}{2 r \sqrt{r(r \pm z)}}\left(\begin{array}{c}
r \pm z \\
\pm(x+i y)
\end{array}\right)
\end{aligned}
$$

to the Freund equations, where $r=\sqrt{x^{2}+y^{2}+z^{2}}$. A monopole solution of this kind was first obtained in [15] (see also [5], where the authors discuss in detail how the Freund equations are related to the SWE on $\mathbb{R}^{3}$ ). 
Example 2. Starting with $\boldsymbol{H}_{(0)}= \pm(\sinh \kappa y, 0,0)$, we obtain the solution ${ }^{5}$

$$
\begin{aligned}
\boldsymbol{B} & =\mp \frac{\kappa^{2}}{\sinh ^{2} \kappa y} \mathbf{e}_{x}, \\
\boldsymbol{A} & = \pm \kappa \operatorname{coth} \kappa y \mathbf{e}_{z}, \\
|\psi\rangle & =\frac{\kappa}{\sqrt{2} \sinh \kappa y}\left(\begin{array}{c}
1 \\
\mp 1
\end{array}\right)
\end{aligned}
$$

to the Seiberg-Witten equations. This solution is essentially the same as the effectively one-dimensional solution to the SWE found in [6]. On the other hand, if we start with $\boldsymbol{H}_{(0)}= \pm(\cosh \kappa y, 0,0)$, we obtain the solution

$$
\begin{aligned}
\boldsymbol{B} & = \pm \frac{\kappa^{2}}{\cosh ^{2} \kappa y} \mathbf{e}_{x} \\
\boldsymbol{A} & = \pm \kappa \tanh \kappa y \mathbf{e}_{z} \\
|\psi\rangle & =\frac{\kappa}{\sqrt{2} \cosh \kappa y}\left(\begin{array}{c}
1 \\
\mp 1
\end{array}\right)
\end{aligned}
$$

to the Freund equations. We note that similar expressions were also obtained in [6] through analytic continuation of the aforementioned one-dimensional solution to the SWE.

Example 3. Starting with $\boldsymbol{H}_{(0)}= \pm(x y z, 0,0)$, we obtain the solution

$$
\begin{aligned}
\boldsymbol{B} & =\mp\left(\frac{1}{y^{2}}+\frac{1}{z^{2}}, 0,0\right), \\
\boldsymbol{A} & = \pm \frac{1}{y^{2}+z^{2}}\left(0,-\frac{y^{2}}{z}, \frac{z^{2}}{y}\right), \\
|\psi\rangle & =\sqrt{\frac{1}{2 y^{2}}+\frac{1}{2 z^{2}}}\left(\begin{array}{c}
1 \\
\mp 1
\end{array}\right)
\end{aligned}
$$

to the Seiberg-Witten equations.

For solutions of this kind, in which $\boldsymbol{B}(\boldsymbol{r})$ is always parallel to some fixed vector $\mathbf{n}$ and only depends on coordinates $(u, v)$ of a plane orthogonal to $\mathbf{n}$, the quantity $\omega=\frac{1}{2} \ln B$ is known [] $]$ to satisfy the Liouville equation $4 \partial_{z} \partial_{\bar{z}} \omega=e^{2 \omega}$, where $z$ now denotes the complex coordinate $z=u+i v$ and $B=\|\boldsymbol{B}\|{ }^{6}{ }^{6}$ Using the ansatz

$$
\omega=\frac{1}{2} \ln \frac{4(d g / d z)(d \bar{g} / d \bar{z})}{(1-g \bar{g})^{2}}
$$

with $g(z)$ an arbitrary analytic function, the authors of [6] construct a family of effectively two-dimensional solutions to the SWE with interesting properties. We note, however, that the above solution (18a) apparently does not belong to such family, obtained via ansatz (19). In any case, we show in the Appendix that the alternative ansatz ${ }^{7}$

$$
\omega=\frac{1}{2} \ln \frac{(d g / d z)(d \bar{g} / d \bar{z})}{[\Im(g)]^{2}},
$$

where $\Im(g)$ denotes the imaginary part of $g$, does yield the above solution. In fact, as discussed in the Appendix, (18a) is the $n=2$ case of a family of two-dimensional singular solutions generated by the choice $g(z)=z^{n}$ in (20), with $n=\frac{1}{2}, 1, \frac{3}{2}, \ldots$.

\footnotetext{
${ }^{5}$ In order to avoid dealing with the absolute value function in the algebraic computation procedure of Examples 2 and 3 , it is useful to first restrict attention to the domain given by $x>0, y>0$, and $z>0$, and later extend the obtained solution to any nonzero $x$, $y$, and $z$.

${ }^{6}$ It should be clear from the context when $z$ refers to the complex coordinate $z=u+i v$ or to the Cartesian coordinate in $(x, y, z)$.

${ }^{7}$ It should be noted that both (19) and 20] are particular cases of the well-known general solution of the Liouville equation [16] (see Appendix).
} 
Example 4. Starting with $\boldsymbol{H}_{(0)}= \pm(y,-x, 0)$, we obtain the axisymmetric solution

$$
\begin{aligned}
\boldsymbol{B} & = \pm \frac{1}{2 \rho^{2}} \mathbf{e}_{\phi} \\
\boldsymbol{A} & =-\frac{1}{2 \rho} \mathbf{e}_{\phi} \pm \frac{1}{2 \rho} \mathbf{e}_{z} \\
|\psi\rangle & =\frac{1}{2 \rho}\left(\begin{array}{c}
1 \\
\pm i e^{i \phi}
\end{array}\right)
\end{aligned}
$$

to the Seiberg-Witten equations, where cylindrical coordinates $\rho, \phi, z$, with $\rho=\sqrt{x^{2}+y^{2}}$ and $\phi=\arctan (y / x)$, were used. ${ }^{8}$ The integral curves of $\boldsymbol{A}$ are helices of constant $\rho$ going upward (downward) with respect to the $z$ axis. We note that the first term $\boldsymbol{A}_{A B}=-\frac{1}{2 \rho} \mathbf{e}_{\phi}$ of $\boldsymbol{A}$ is in fact an Aharonov-Bohm potential, with holonomy given by

$$
\int_{\gamma} \boldsymbol{A}_{A B}(\boldsymbol{r}) \cdot d \boldsymbol{r}=-\pi
$$

where $\gamma$ is any loop winding once around the $z$ axis. Note that the Aharonov-Bohm term $\boldsymbol{A}_{A B}$ is actually implied by $\boldsymbol{B}$ (through Eq. (15b)) even though $\boldsymbol{B}$ in Eq. (21a) does not receive any contribution from the curl of $\boldsymbol{A}_{A B}$ (which is actually zero for $\rho \neq 0$ ). In this way, the purely azimuthal magnetic field of Eq. (21a), defined away from the $z$ axis, unavoidably gives rise, through $\boldsymbol{A}_{A B}$, to the "additional" singular magnetic field $-\pi \delta(x) \delta(y) \mathbf{e}_{z}$ along the $z$ axis (which is the same as the magnetic field of an infinitely long and infinitesimally thin solenoid at the $z$ axis).

It is also interesting to note that, although the two solutions in Eq. 21a (given, respectively, by its plus and minus signs) wind in opposite directions with respect to the $x y$ plane, their associated potentials both wind clockwise, with identical Aharonov-Bohm terms, the only difference residing in their $z$ components.

\section{CLOSING REMARKS}

In all the examples above, the final solution for $\boldsymbol{H}$ is obtained in exact form after very few iterations. On the other hand, some experience with the above computational experiment shows that a generic initial condition for $\boldsymbol{H}$ typically leads to an increasingly complicated algebraic expression at each iteration, thereby requiring further investigation on convergence issues related to such method. A natural question to ask is what are the initial conditions under which the sequence $\boldsymbol{H}_{(k)}$ may be guaranteed to converge since, under such circumstances, the approach presented here could be used to define classes of solutions to the SWE and Freund equations iteratively.

Finally, we note that the approach presented here suggests a natural generalization to the four-dimensional case, where one may try to lift the whole set of (Euclidean) Maxwell equations to obtain (singular) solutions to the SWE on $\mathbb{R}^{4}$. This is the subject of work in progress.

\section{Acknowledgments}

The author is grateful to C. Adam, W. A. Rodrigues Jr., M. A. F. Rosa, A. Saa and J. Vaz Jr. for helpful discussions, and to the Abdus Salam International Centre for Theoretical Physics, Trieste, Italy, where this work was partly done, for hospitality. This work was supported by FAPESP.

\section{APPENDIX}

The general solution to the Liouville equation,

$$
4 \partial_{z} \partial_{\bar{z}} \omega=e^{2 \omega}
$$

\footnotetext{
${ }^{8}$ Such $\boldsymbol{B}$ is similar (but different in the $\rho$-dependence) to the magnetic field $\boldsymbol{B} \propto \frac{1}{\rho} \mathbf{e}_{\phi}$ produced by a steady current along the $z$ axis.
} 
was first given by Liouville in [16] (where the author considers, in fact, the corresponding equation for $\lambda=e^{2 \omega}$, and with real variables). It is given by

$$
\omega(z, \bar{z})=\frac{1}{2} \ln \frac{4(d g(z) / d z)(d h(\bar{z}) / d \bar{z})}{[1-g(z) h(\bar{z})]^{2}}
$$

where $g(z)$ and $h(\bar{z})$ are arbitrary analytic and anti-analytic functions, respectively. The ansatz of [6], given by Eq. (19), is recovered from Eq. (23) if the natural choice $h(\bar{z})=\overline{g(z)}$ is made. However, as mentioned in Example 3 of Section III the solution given by $B=1 / u^{2}+1 / v^{2}$ is apparently not recovered by such ansatz (recall that $B$ is related to $\omega$ by $B=e^{2 \omega}$, where $B=\|\boldsymbol{B}\|$ and now $z$ denotes the complex coordinate $\left.z=u+i v\right)$. This motivates the search for an alternative ansatz for such $B$. Generalizing the above choice of $h$ in terms of $g$ to $h(\bar{z})=\overline{g(z)^{\nu}}$, it is not hard to show that, in general, the requirement that $\omega$ be real restricts $\nu$ to +1 or -1 , which yield Eq. (19) and Eq. (20), respectively. Therefore, the alternative ansatz (20) is given by the choice $h(\bar{z})=1 / \overline{g(z)}$ in $(23)$. The solution of Example 3 is recovered from such ansatz for $g(z)=z^{2}$, as a direct calculation shows.

More generally, choosing $g(z)=z^{n}$ in Eq. (20) leads to the solution $B=\frac{n^{2}}{\rho^{2} \sin ^{2} n \phi}$, where polar coordinates $z=\rho e^{i \phi}$ were used. The requirement that $B$ be single-valued restricts $n$ to $n=\frac{1}{2}, 1, \frac{3}{2}, \ldots$ (note that $B$ is insensitive to the change $n \rightarrow-n$ ). In this way, the solution of Example 3 is the $n=2$ case of such family of two-dimensional singular solutions. Note that the solution corresponding to a given $n$ is singular along $2 n$ lines starting at the origin and passing through the roots of unity of order $2 n$.

[1] P. Kustaanheimo and E. Stiefel, J. Reine Angew. Math. 218, 204 (1965).

[2] M. Kibler, in Group Theoretical Methods in Physics, H. D. Doebner et al (eds.), Springer-Verlag, Berlin: Lecture Notes in Physics 313, p. 238 (1988).

[3] T. Bartsch, J. Phys. A 36, 6963 (2003), physics/0301017

[4] E. Witten, Math. Res. Lett. 1, 769 (1994), hep-th/9411102

[5] C. Adam, B. Muratori and C. Nash, J. Math. Phys. 41, 5875 (2000), hep-th/0003125

[6] S. Nergiz and C. Saçlıog̃lu, J. Math. Phys. 37, 3753 (1996), hep-th/9602088

[7] M. Loss and H. Yau, Commun. Math. Phys. 104, 283 (1986).

[8] D. V. Aleynikov and E. A. Tolkachev (2002), hep-th/0206211

[9] See, e.g., E. Merzbacher, Quantum Mechanics, 2nd edition, Wiley (1970).

[10] For a comprehensive overview of diverse appearances of the (first) Hopf fibration in Physics, see H. K. Urbantke, J. Geom. Phys. 46, 125 (2003).

[11] J. P. Crawford, J. Math. Phys. 26, 1439 (1985).

[12] J. Vaz Jr., in Clifford algebras and their application in mathematical physics, V. Dietrich et al (eds.), Kluwer, Dordrecht, p. 401 (1998).

[13] R. A. Mosna and J. Vaz Jr., Phys. Lett. A 315, 418 (2003), quant-ph/0303072

[14] D. V. Aleynikov and E. A. Tolkachev, J. Phys. A 36, 2251 (2003).

[15] P. G. O. Freund, J. Math. Phys. 36, 2673 (1995), hep-th/9412208

[16] J. Liouville, J. Math. Pure Appl. 18, 71 (1853). 